\title{
On level one cuspidal Bianchi modular forms
}

\author{
Alexander D. Rahm and Mehmet Haluk Şengün
}

\begin{abstract}
In this paper, we present the outcome of vast computer calculations, locating several of the very rare instances of level one cuspidal Bianchi modular forms that are not lifts of elliptic modular forms.
\end{abstract}

\section{Introduction}

Bianchi modular forms over an imaginary quadratic field $K$ are automorphic forms of cohomological type associated to the $\mathbb{Q}$-algebraic group $\operatorname{Res}_{K / \mathbb{Q}}\left(\mathrm{SL}_{2}\right)$. Even though modern studies of Bianchi modular forms go back to the mid 1960s, most of the fundamental problems surrounding their theory are still wide open. In this paper, we report on our extensive computations that show the paucity of genuine level one cuspidal Bianchi modular forms.

Let $S_{k}(1)$ denote the space of level one weight $k+2$ cuspidal Bianchi modular forms over $K=\mathbb{Q}(\sqrt{-d})$. In their recent paper $[\mathbf{1 4}]$, Finis, Grunewald and Tirao computed the dimension of the subspace $L_{k}(1)$ of $S_{k}(1)$ which is formed by (twists of) those forms which arise from elliptic cuspidal modular forms via base-change or arise from a quadratic extension of $K$ via automorphic induction (see [14] for these notions). In this paper, we investigate numerically how much of $S_{k}(1)$ is exhausted by $L_{k}(1)$. There have been previous reports, however of limited size, in the 2009 paper [8] of Calegari and Mazur (the computations in that paper were carried out by Pollack and Stein) and in the 2010 paper [14] of Finis, Grunewald and Tirao. While the computations in [8] were limited to the case $d=2$, the computations in [14] covered ten imaginary quadratic fields. The precise scope of the computations in [14] is given in Table 1 below.

It was observed in [8] that for $2 k \leqslant 96$, one has $L_{2 k}(1)=S_{2 k}(1)$. The computations of [14] extended those of [8]. An interesting outcome of the data they collected is that, except in two of the 946 spaces they computed, one has $L_{k}(1)=S_{k}(1)$. The exceptional cases are $(d, k)=(7,12)$ and $(d, k)=(11,10)$. In both cases, there is a two-dimensional complement to $L_{k}(1)$ inside $S_{k}(1)$.

Using a different and more efficient approach, we computed, over more than 800 processordays, the dimension of 4986 different spaces $S_{k}(1)$ over 186 different imaginary quadratic fields. The precise scope of our computations is given in Tables 2 and 3, where $D$ and $h$ denote the discriminant and the class number of $K$ respectively. In only 29 of these spaces were we able to observe genuine forms. The precise data about these exceptional cases is provided in Table 4.

TABLE 1. Finis-Grunewald-Tirao test range.

\begin{tabular}{lcccccccccc}
\hline$d$ & 1 & 2 & 3 & 7 & 11 & 19 & 5 & 6 & 10 & 14 \\
\hline$k \leqslant$ & 104 & 141 & 116 & 132 & 153 & 60 & 60 & 60 & 60 & 60 \\
\hline
\end{tabular}

Received 10 September 2012; revised 19 February 2013.

2010 Mathematics Subject Classification 11F55, 11F75 (primary), 57T15 (secondary).

The first author is funded by the Irish Research Council. The second author is funded by a Marie Curie Intra-European Fellowship. 
In Section 2, we briefly discuss the conjectural connections between the spaces $S_{0}(1)$ and Abelian varieties defined over $K$ of $\mathrm{GL}_{2}$-type. In Section 3, we make some speculations in light of the data we collect. In particular, we pose a question which can be seen as a Bianchi modular forms analogue of Maeda's conjecture. Finally in Section 4, we explain how we carried out our computations. As usual, the starting point of our approach is the so called 'Eichler-ShimuraHarder' isomorphism, which allows us to replace the calculation of $S_{k}(1)$ by the calculation of the cohomology of the relevant Bianchi group with special non-trivial coefficients. Then, to compute this cohomology space, we use the program Bianchi.gp [23], which analyzes the structure of the Bianchi group via its action on hyperbolic 3-space (which is isomorphic to the associated symmetric space $\left.\mathrm{SL}_{2}(\mathbb{C}) / \mathrm{SU}_{2}\right)$. We then feed this group-geometric information into an equivariant spectral sequence that gives us an explicit description of the second degree cohomology of the Bianchi group, with the relevant coefficients.

TABLE 2. The scope of our computations, part 1.

\begin{tabular}{|c|c|c|c|c|c|c|c|c|c|c|c|}
\hline$|D|$ & 3 & 4 & 7 & 8 & 11 & 15 & 19 & 20 & 23 & 24 & 31 \\
\hline$h$ & 1 & 1 & 1 & 1 & 1 & 2 & 1 & 2 & 3 & 2 & 3 \\
\hline$k \leqslant$ & 219 & 216 & 217 & 217 & 217 & 115 & 120 & 100 & 83 & 101 & 74 \\
\hline$|D|$ & 35 & 39 & 40 & 43 & 47 & 51 & 52 & 55 & 56 & 59 & 67 \\
\hline$h$ & 2 & 4 & 2 & 1 & 5 & 2 & 2 & 4 & 4 & 3 & 1 \\
\hline$k \leqslant$ & 86 & 67 & 73 & 83 & 52 & 75 & 65 & 45 & 55 & 60 & 58 \\
\hline$|D|$ & 68 & 71 & 79 & 83 & 84 & 87 & 88 & 91 & 95 & 103 & 104 \\
\hline$h$ & 4 & 7 & 5 & 3 & 4 & 6 & 2 & 2 & 8 & 5 & 6 \\
\hline$k \leqslant$ & 53 & 38 & 33 & 41 & 50 & 36 & 45 & 50 & 30 & 30 & 32 \\
\hline$|D|$ & 107 & 111 & 115 & 116 & 119 & 120 & 123 & 127 & 131 & 132 & 136 \\
\hline$h$ & 3 & 8 & 2 & 6 & 10 & 4 & 2 & 5 & 5 & 4 & 4 \\
\hline$k \leqslant$ & 35 & 28 & 40 & 33 & 25 & 38 & 35 & 25 & 32 & 33 & 32 \\
\hline$|D|$ & 139 & 143 & 148 & 151 & 152 & 155 & 159 & 163 & 164 & 167 & 168 \\
\hline$h$ & 3 & 10 & 2 & 7 & 6 & 4 & 10 & 1 & 8 & 11 & 4 \\
\hline$k \leqslant$ & 29 & 20 & 31 & 21 & 24 & 26 & 19 & 33 & 24 & 18 & 26 \\
\hline$|D|$ & 179 & 183 & 184 & 187 & 191 & 195 & 199 & 203 & 211 & 212 & 215 \\
\hline$h$ & 5 & 8 & 4 & 2 & 13 & 4 & 9 & 4 & 3 & 6 & 14 \\
\hline$k \leqslant$ & 24 & 19 & 25 & 25 & 15 & 27 & 17 & 23 & 21 & 17 & 14 \\
\hline$|D|$ & 219 & 223 & 227 & 228 & 231 & 232 & 235 & 239 & 244 & 247 & 248 \\
\hline$h$ & 4 & 7 & 5 & 4 & 12 & 2 & 2 & 15 & 6 & 6 & 8 \\
\hline$k \leqslant$ & 20 & 14 & 17 & 18 & 13 & 21 & 23 & 12 & 17 & 13 & 16 \\
\hline$|D|$ & 251 & 255 & 259 & 260 & 263 & 264 & 267 & 271 & 276 & 280 & 283 \\
\hline$h$ & 7 & 12 & 4 & 8 & 13 & 8 & 2 & 11 & 8 & 4 & 3 \\
\hline$k \leqslant$ & 15 & 14 & 17 & 14 & 13 & 15 & 21 & 12 & 16 & 16 & 17 \\
\hline$|D|$ & 287 & 291 & 292 & 295 & 296 & 299 & 303 & 307 & 308 & 311 & 312 \\
\hline$h$ & 14 & 4 & 4 & 8 & 10 & 8 & 10 & 3 & 8 & 19 & 4 \\
\hline$k \leqslant$ & 12 & 19 & 16 & 13 & 13 & 13 & 11 & 15 & 13 & 11 & 13 \\
\hline$|D|$ & 319 & 323 & 327 & 328 & 331 & 335 & 339 & 340 & 344 & 347 & 355 \\
\hline$h$ & 10 & 4 & 12 & 4 & 3 & 18 & 6 & 4 & 10 & 5 & 4 \\
\hline$k \leqslant$ & 11 & 12 & 10 & 13 & 14 & 11 & 15 & 14 & 10 & 12 & 13 \\
\hline$|D|$ & 356 & 359 & 367 & 371 & 372 & 376 & 379 & 383 & 388 & 391 & 395 \\
\hline$h$ & 12 & 19 & 9 & 8 & 4 & 8 & 3 & 17 & 4 & 14 & 8 \\
\hline$k \leqslant$ & 11 & 9 & 11 & 10 & 12 & 12 & 13 & 8 & 11 & 9 & 10 \\
\hline$|D|$ & 399 & 403 & 404 & 407 & 408 & 411 & 415 & 419 & 420 & 424 & 427 \\
\hline$h$ & 16 & 2 & 14 & 16 & 4 & 6 & 10 & 9 & 8 & 6 & 2 \\
\hline$k \leqslant$ & 8 & 12 & 9 & 8 & 10 & 12 & 10 & 12 & 11 & 10 & 13 \\
\hline
\end{tabular}




\section{Background}

Let $K$ be an imaginary quadratic field with ring of integers $\mathcal{O}$. Let $\Gamma$ be the Bianchi group $\mathrm{SL}_{2}(\mathcal{O})$. It is a discrete subgroup of the real Lie group $\mathrm{SL}_{2}(\mathbb{C})$ and thus acts discontinuously on hyperbolic 3-space. Let $Y_{\Gamma}$ be the quotient hyperbolic 3-fold. The Borel-Serre compactification $X_{\Gamma}$ of $Y_{\Gamma}$, see [31, appendix], is a compact 3-fold with boundary $\partial X_{\Gamma}$ and with interior homeomorphic to $Y_{\Gamma}$. When the discriminant of $K$ is smaller than $-4, \partial X_{\Gamma}$ consists of $h_{K}$ disjoint 2-tori, where $h_{K}$ is the class number of $K$.

Given $n \geqslant 0$, let $\mathbb{C}[x, y]_{n}$ denote the space of homogeneous polynomials of degree $n$ on variables $x, y$ with complex coefficients; $\mathrm{SL}_{2}(\mathbb{C})$ acts on this space in the obvious way permitted by the two variables. Consider the $\mathrm{SL}_{2}(\mathbb{C})$-module

$$
E_{n}:=\mathbb{C}[x, y]_{n} \otimes_{\mathbb{C}}{\overline{\mathbb{C}}[x, y]_{n}}_{n}
$$

where the overline on the second factor is to indicate that the action on this factor is twisted with complex conjugation. When considered as a $\Gamma$-module, $E_{n}$ gives rise to a locally constant

TABLE 3. The scope of our computations, part 2.

\begin{tabular}{|c|c|c|c|c|c|c|c|c|c|}
\hline$|D|$ & 431 & 435 & 436 & 439 & 440 & 443 & 447 & 451 & 452 \\
\hline$h$ & 21 & 4 & 6 & 15 & 12 & 5 & 14 & 6 & 8 \\
\hline$k \leqslant$ & 8 & 13 & 11 & 9 & 8 & 12 & 10 & 12 & 11 \\
\hline$|D|$ & 455 & 456 & 463 & 467 & 471 & 472 & 479 & 483 & 487 \\
\hline$h$ & 20 & 8 & 7 & 7 & 16 & 6 & 25 & 4 & 7 \\
\hline$k \leqslant$ & 7 & 10 & 8 & 10 & 7 & 11 & 7 & 11 & 8 \\
\hline$|D|$ & 488 & 491 & 499 & 520 & 532 & 547 & 555 & 560 & 568 \\
\hline$h$ & 10 & 9 & 3 & 4 & 4 & 3 & 4 & 4 & 4 \\
\hline$k \leqslant$ & 9 & 10 & 10 & 9 & 10 & 11 & 11 & 7 & 10 \\
\hline$|D|$ & 571 & 595 & 627 & 643 & 667 & 696 & 708 & 715 & 723 \\
\hline$h$ & 5 & 4 & 4 & 3 & 4 & 12 & 4 & 4 & 4 \\
\hline$k \leqslant$ & 11 & 9 & 11 & 9 & 9 & 4 & 7 & 7 & 9 \\
\hline$|D|$ & 760 & 763 & 795 & 883 & 907 & 955 & 1003 & 1027 & 1051 \\
\hline$h$ & 4 & 4 & 4 & 3 & 3 & 4 & 4 & 4 & 5 \\
\hline$k \leqslant$ & 7 & 7 & 7 & 6 & 7 & 6 & 6 & 5 & 5 \\
\hline$|D|$ & 1123 & 1227 & 1243 & 1387 & 1411 & 1507 & 1555 & 1723 & 1747 \\
\hline$h$ & 5 & 4 & 4 & 4 & 4 & 4 & 4 & 5 & 5 \\
\hline$k \leqslant$ & 4 & 5 & 4 & 4 & 4 & 4 & 4 & 3 & 3 \\
\hline$|D|$ & 1867 & & & & & & & & \\
\hline$h$ & 5 & & & & & & & & \\
\hline$k \leqslant$ & 3 & & & & & & & & \\
\hline
\end{tabular}

TABLE 4. The cases where there are genuine classes.

\begin{tabular}{lcccccccccc}
\hline$D \mid$ & $\mathbf{7}$ & $\mathbf{1 1}$ & $\mathbf{7 1}$ & $\mathbf{8 7}$ & $\mathbf{9 1}$ & $\mathbf{1 5 5}$ & $\mathbf{1 9 9}$ & $\mathbf{2 2 3}$ & $\mathbf{2 3 1}$ & $\mathbf{3 3 9}$ \\
$k$ & 12 & 10 & 1 & 2 & 6 & 4 & 1 & 0 & 4 & 1 \\
$\operatorname{dim}$ & 2 & 2 & 2 & 2 & 2 & 2 & 4 & 2 & 2 & 2 \\
$|D|$ & $\mathbf{3 4 4}$ & $\mathbf{4 0 7}$ & $\mathbf{4 0 8}$ & $\mathbf{4 0 8}$ & $\mathbf{4 0 8}$ & $\mathbf{4 1 5}$ & $\mathbf{4 3 5}$ & $\mathbf{4 3 5}$ & $\mathbf{4 3 5}$ & $\mathbf{4 3 5}$ \\
$k$ & 1 & 0 & 2 & 5 & 8 & 0 & 2 & 5 & 8 & 11 \\
$\operatorname{dim}$ & 2 & 2 & 2 & 2 & 2 & 2 & 2 & 2 & 2 & 2 \\
$|D|$ & $\mathbf{4 5 5}$ & $\mathbf{4 8 3}$ & $\mathbf{5 7 1}$ & $\mathbf{5 7 1}$ & $\mathbf{6 4 3}$ & $\mathbf{7 6 0}$ & $\mathbf{1 0 0 3}$ & $\mathbf{1 0 0 3}$ & $\mathbf{1 0 5 1}$ & \\
$k$ & 0 & 1 & 0 & 1 & 0 & 2 & 0 & 1 & 0 & \\
$\operatorname{dim}$ & 2 & 2 & 2 & 2 & 2 & 2 & 2 & 2 & 2 & \\
\hline
\end{tabular}


sheaf $\mathcal{E}_{n}$ on $Y_{\Gamma}$ whose stalks are isomorphic to $E_{n}$. Consider the long exact sequence

$$
\ldots \rightarrow H_{c}^{i}\left(Y_{\Gamma} ; \mathcal{E}_{n}\right) \rightarrow H^{i}\left(X_{\Gamma} ; \overline{\mathcal{E}}_{n}\right) \rightarrow H^{i}\left(\partial X_{\Gamma} ; \overline{\mathcal{E}}_{n}\right) \rightarrow \ldots,
$$

where $H_{c}^{i}$ denotes the compactly supported cohomology and $\overline{\mathcal{E}}_{n}$ is a certain natural extension of $\mathcal{E}_{n}$ to $X_{\Gamma}$.

The cuspidal cohomology $H_{\text {cusp }}^{i}$ is defined to be the image of the compactly supported cohomology. The Eisenstein cohomology $H_{\mathrm{Eis}}^{i}$ is the complement of the cuspidal cohomology inside $H^{i}$ and it is isomorphic to the image of the restriction map inside the cohomology of the boundary. The decomposition $H^{i}=H_{\text {cusp }}^{i} \oplus H_{\text {Eis }}^{i}$ respects the Hecke action which is defined, as usual, via correspondences on $X_{\Gamma}$.

By construction, the embedding $Y_{\Gamma} \hookrightarrow X_{\Gamma}$ is a homotopy invariance. Together with the fact that $Y_{\Gamma}$ is a $K(\Gamma, 1)$-space, we get the isomorphisms

$$
H^{i}\left(X_{\Gamma} ; \overline{\mathcal{E}}_{n}\right) \simeq H^{i}\left(Y_{\Gamma} ; \mathcal{E}_{n}\right) \simeq H^{i}\left(\Gamma ; E_{n}\right)
$$

Via these isomorphisms, we define the cuspidal and Eisenstein parts of $H^{i}\left(\Gamma ; E_{n}\right)$.

Let $S_{n}(1)$ denote the space of level one cuspidal Bianchi modular forms (over $K$ ) of weight $n+2$. It is well known that

$$
S_{n}(1) \simeq H_{\mathrm{cusp}}^{1}\left(Y_{\Gamma} ; \mathcal{E}_{n}\right) \simeq H_{\mathrm{cusp}}^{2}\left(Y_{\Gamma} ; \mathcal{E}_{n}\right)
$$

as Hecke modules. Here the first isomorphism was established by Harder and the second follows from duality, see [3].

In [14], a formula for the dimension of the space $L_{n}(1)$ has been given for all fields $K$ and weights $n$. We will compare the dimension of $L_{n}(1)$, which we obtain via that formula, with the dimension of $S_{n}(1)$, which we will obtain via our computer programs. The following proposition will allow us to deduce the size of the cuspidal cohomology, and hence of $S_{n}(1)$, once we have computed the size of the whole cohomology. It is well known to specialists, however for the convenience of the reader we include a proof of it.

Proposition 1. Let $K$ be an imaginary quadratic field. Then in the above notation

$$
\operatorname{dim} H_{\text {Eis }}^{2}\left(X_{\Gamma} ; \overline{\mathcal{E}}_{n}\right)= \begin{cases}h_{K}-1 & \text { if } n=0, \\ h_{K} & \text { otherwise }\end{cases}
$$

where $h_{K}$ is the class number of $K$.

Proof. It is well known (see [17, Theorem 2.1]) that the map

$$
H^{2}\left(X_{\Gamma} ; \overline{\mathcal{E}}_{n}\right) \longrightarrow H^{2}\left(\partial X_{\Gamma} ; \overline{\mathcal{E}}_{n}\right)
$$

is surjective for $n>0$ and its image has codimension one for $n=0$.

Assume that the discriminant of $K$ is less than -4 , that is, $K$ is not equal to $\mathbb{Q}(i)$ nor $\mathbb{Q}(\sqrt{-3})$. Then the boundary $\partial X_{\Gamma}$ is a disjoint union of 2-tori, indexed by the class group of $K$. Below we prove that for every boundary component $T$ of $\partial X_{\Gamma}$, the dimension of $H^{2}\left(T ; \overline{\mathcal{E}}_{n}\right)$ is 1 , which clearly yields our claim.

Let $c \in K \cup\{\infty\}$ be a cusp and let $\Gamma_{c}$ be its stabilizer in $\Gamma$ (which is a parabolic subgroup). Then $\Gamma_{c}$ is the fundamental group of the 2-torus $T_{c}$ in $\partial X_{\Gamma}$. In fact, $T_{c}$ is a $K\left(\Gamma_{c}, 1\right)$-space. Hence we can turn our attention to computing $H^{2}\left(\Gamma_{c} ; E_{n}\right)$. Composition of the cup product and the well-known perfect pairing $(\cdot, \cdot): E_{n} \otimes_{\mathbb{C}} E_{n} \rightarrow \mathbb{C}$ (see, for example, $[4, \S 2.4]$ ) gives us 
a pairing as follows.

$$
\begin{array}{r}
H^{0}\left(\Gamma_{c} ; E_{n}\right) \times H^{2}\left(\Gamma_{c} ; E_{n}\right) \stackrel{\cup}{\longrightarrow} H^{2}\left(\Gamma_{c} ; E_{n} \otimes_{\mathbb{C}} E_{n}\right) \\
\downarrow(\cdot, \cdot) \\
H^{2}\left(\Gamma_{c} ; \mathbb{C}\right) \simeq \mathbb{C}
\end{array}
$$

Here the last isomorphism follows from the fact that $T_{c}$ is a compact 2-fold (see also [29, Proof of Proposition 3.5] for a direct algebraic argument). Thus the dimension we are looking for is equal to that of $H^{0}\left(\Gamma_{c} ; E_{n}\right)$. Clearly, if $n=0$, the latter dimension is one and thus the dimension of $H^{2}\left(\partial X_{\Gamma} ; \overline{\mathcal{E}}_{n}\right)$ is $h_{K}$, as desired.

Let us now assume that $n \neq 0$. Conjugation by a matrix in $\mathrm{SL}_{2}(K)$ which takes $c$ to the cusp at infinity induces an isomorphism

$$
\Gamma_{c} \simeq \Gamma_{\infty}=\left(\begin{array}{cc}
* & * \\
0 & *
\end{array}\right) \subset \mathrm{SL}_{2}\left(\mathcal{O}_{K}\right)
$$

Consider the normal subgroup $\Gamma_{\infty}^{+}:=\left(\begin{array}{ll}1 & * \\ 0 & 1\end{array}\right)$ of $\Gamma_{\infty}$. Then $\Gamma_{\infty}^{+}$is a free Abelian group on two generators. We are now going to determine the submodule $E_{n}^{\Gamma_{\infty}^{+}}$of $E_{n}$ invariant under its action. As the generators are of the form $\left(\begin{array}{ll}1 & * \\ 0 & 1\end{array}\right)$, it is clear that the vector $x^{n} \otimes x^{n}$ is fixed by $\Gamma_{\infty}^{+}$. One shows, proceeding as in [35, Lemma 2.4], that there are no other fixed vectors. Hence

$$
H^{0}\left(\Gamma_{\infty}^{+} ; E_{n}\right)=E_{n}^{\Gamma_{\infty}^{+}}=\left\langle x^{n} \otimes x^{n}\right\rangle
$$

is of complex dimension one. Let $\mu:=\Gamma_{\infty} / \Gamma_{\infty}^{+}=\left\{\left(\begin{array}{cc} \pm 1 & 0 \\ 0 & \pm 1\end{array}\right)\right\}$. As we are considering modules over $\mathbb{C}$, it follows that

$$
H^{0}\left(\Gamma_{\infty} ; E_{n}\right) \simeq H^{0}\left(\Gamma_{\infty}^{+} ; E_{n}\right)^{\mu}
$$

is the invariant submodule under $\mu$. We easily check that the action of $\mu$ on $E_{n}$ is trivial, and so

$$
H^{0}\left(\Gamma_{c} ; E_{n}\right) \simeq H^{0}\left(\Gamma_{\infty}^{+} ; E_{n}\right)
$$

is again of complex dimension one. This completes the proof with our assumption on the discriminant of $K$.

When $K$ is $\mathbb{Q}(i)$ or $\mathbb{Q}(\sqrt{-3})$, due to the extra units, the cross-sections of the cusps, which are again parametrized by the class group, are 2-orbifolds whose underlying manifolds are 2spheres (torus folded by an involution). As the second degree cohomology of the 2-sphere is one dimensional, the result follows.

\section{Abelian varieties of $\mathrm{GL}(2)$-type}

There is a widely believed conjectural connection between Bianchi newforms of weight two over $K$ and Abelian varieties of $\mathrm{GL}_{2}$-type defined over $K$ (see $[\mathbf{1 0}, \mathbf{1 3}, \mathbf{3 3}]$ ) which is expressed in terms of the associated $L$-functions. In particular, an Abelian variety of $\mathrm{GL}_{2}$-type over $K$ that is not definable over $\mathbb{Q}$ nor of $C M$-type, with everywhere good reduction, is expected to give rise to newforms in $S_{0}(1)^{+}$. Here $S_{0}(1)^{+}$denotes the plus-subspace of $S_{0}(1)$ in the sense of $[\mathbf{9}, \mathbf{1 3}]$. Equivalently, $S_{0}(1)^{+}$can be seen as the space of cuspidal Bianchi modular forms of weight two for $\mathrm{GL}_{2}\left(\mathcal{O}_{K}\right)$.

In the reverse direction, the newforms in $S_{0}(1)^{+}$are expected ${ }^{\dagger}$ to correspond to Abelian varieties of $\mathrm{GL}_{2}$-type over $K$ which have everywhere good reduction. As listed in Table 4,

\footnotetext{
${ }^{\dagger}$ There are some natural exceptions coming from elliptic newforms with inner twists, see Cremona [10], which are avoided if we consider newforms that are not in $L_{0}(1)$.
} 
we have found eight imaginary quadratic fields for which $S_{0}(1)$ contained non-lifted classes. For only six of these fields, the non-lifted classes were in fact contained in $S_{0}(1)^{+}$. In Table 5 below, we list the (necessarily totally real) number field $F$ generated by the Hecke eigenvalues of the non-lifted newforms in these six cases.

We have computed these fields using Dan Yasaki's program, see [36], in Magma which computes the Hecke action on $S_{0}\left(\Gamma_{0}(\mathfrak{n})\right)^{+}$for congruence subgroups of type $\Gamma_{0}(\mathfrak{n})$ of Bianchi groups. Note that as this program only treats $\mathrm{GL}_{2}$-cohomology with trivial weight, that is $k=0$, we could not have used it for our experiment.

Table 5 tells us that there should exist an elliptic curve defined over $\mathbb{Q}(\sqrt{-643})$, and not over $\mathbb{Q}$, which has everywhere good reduction and it should be modular. Indeed, we know by Krämer $[\mathbf{1 9}]$ that there is such an elliptic curve over $\mathbb{Q}(\sqrt{-643})$ and it does seem to be modular, see Scheutzow [28]. Similarly, there should exist Abelian surfaces defined over $\mathbb{Q}(\sqrt{-d})$ with $d=223,415,455,571,1003$ and not over $\mathbb{Q}$, which have everywhere good reduction and real multiplication by $\sqrt{2}, \sqrt{3}, \sqrt{5}, \sqrt{5}, \sqrt{7}$ respectively and they should be modular. Locating such surfaces is a highly non-trivial task, see $[\mathbf{3 0}, \S 8]$.

\section{Comments}

The data collected in this paper make it clear that the spaces of cuspidal Bianchi modular forms of level one are generically made of forms which are not genuine. Unfortunately the data are not enough to formulate a quantitative statement about the occurrences of non-lifted forms. Hence the following question remains.

QUeSTION 2. Let $K$ be an imaginary quadratic field. Let $S_{k}(1)$ denote the space of level one cuspidal Bianchi modular forms over $K$ of weight $k+2$. Is it true that there are at most finitely many $k$ for which the space $S_{k}(1)$ contains non-lifted forms?

Let us make a comparison with other types of modular forms. For the case of Hilbert modular forms (this is the case of the algebraic group $\operatorname{Res}_{F / \mathbb{Q}}\left(\mathrm{SL}_{2}\right)$ where $F$ is a totally real field) and Siegel modular forms of genus 2 (the case of the algebraic group $\mathrm{Sp}_{4}$ ), one has a considerable number of genuine level one cuspidal forms. However, the case of the modular forms for $\mathrm{SL}_{3}$ is similar to our case. Here, in the range of the data collected by Ash and Pollack, see [2], the spaces of level one modular forms for $\mathrm{SL}_{3}$ are completely made of those which are the symmetric square lifts of classical holomorphic modular forms. They in fact conjecture that this is always the case.

It is interesting to note that for the Hilbert and Siegel modular forms of genus 2, where we have plenty of genuine forms, the associated symmetric spaces are Hermitian, while for the Bianchi and $\mathrm{SL}_{3}$ modular forms, where there is an extreme paucity of genuine forms, the associated symmetric spaces fail to be Hermitian. Is this part of a general phenomenon?

Next we shall pose a question about the non-lifted newforms in $S_{k}(1)$ inspired by the classical form of Maeda's conjecture. The non-trivial automorphism $\sigma \in \operatorname{Gal}(K / \mathbb{Q})$ of $K$ acts on the set of newforms in $S_{k}(1)$ as an involution, again denoted $\sigma$. Thus, every newform $f$ has a twin,

TABLE 5. The number field generated by the Hecke eigenvalues of non-lifted newforms in $S_{0}(1)^{+}$.

\begin{tabular}{lcccccc}
\hline$|D|$ & $\mathbf{2 2 3}$ & $\mathbf{4 1 5}$ & $\mathbf{4 5 5}$ & $\mathbf{5 7 1}$ & $\mathbf{6 4 3}$ & $\mathbf{1 0 0 3}$ \\
$F$ & $\mathbb{Q}(\sqrt{2})$ & $\mathbb{Q}(\sqrt{3})$ & $\mathbb{Q}(\sqrt{5})$ & $\mathbb{Q}(\sqrt{5})$ & $\mathbb{Q}$ & $\mathbb{Q}(\sqrt{7})$ \\
\hline
\end{tabular}


denoted ${ }^{\sigma} f$. The Hecke eigenvalues $c(\cdot, \pi)$ associated to the Hecke operators ${ }^{\dagger} T_{\pi}$ satisfy the relation

$$
c\left({ }^{\sigma} f, \pi\right)=c(f, \sigma(\pi))
$$

for every $\pi \in \mathcal{O}$. Recall that just as in the case of elliptic modular forms, for a newform $f$ in $S_{k}(1)$ with Hecke eigenvalue field $F$, there is a newform $f^{\tau}$ in $S_{k}(1)$ for every $\tau \in \operatorname{Gal}(F / \mathbb{Q})$ with the property that $c\left(f^{\tau}, \pi\right)=\tau(c(f, \pi))$ for every $\pi \in \mathcal{O}$. We say that $f$ and the $f^{\tau}$ form one Galois orbit.

QUESTION 3. Let $K$ be an imaginary quadratic field. Is it true that for every $k \geqslant 0$, the set of non-lifted newforms in $S_{k}(1)$, modulo the action of $\operatorname{Gal}(K / \mathbb{Q})$, forms one Galois orbit?

In all except one of the cases where we observed non-lifted newforms, the dimension of the non-lifted subspace was only two. In this case, the answer to Question 3 is automatically yes as the two non-lifted newforms have to be twins (that is, the Galois conjugate of the newform is equal to its twin). Page kindly computed the Hecke action (based on the methods of [21]) on the non-lifted classes for the case $(199,1)$ for us and his data show that the answer to Question 3 is yes in this case as well. More precisely, we have a pair of Galois conjugate non-lifted newforms with coefficients in $\mathbb{Q}(\sqrt{13})$ and their twins, forming the four dimensional non-lifted subspace.

As Calegari remarked to us, if there are two elliptic curves defined over $K$ with good reduction everywhere and such that neither come from $\mathbb{Q}$ nor are conjugates of each other, then the answer to Question 3 would be no for $S_{2}(1)$. Note that the analogue of this conjecture for Hilbert modular forms over real quadratic fields holds in the range of the computations performed by Doi and Ishii, see [12, p. 568].

\section{Method of the computations}

In this section, we will explain how we computed the cohomology of the investigated Bianchi groups.

Let $m$ be a square-free positive integer and $K=\mathbb{Q}(\sqrt{-m})$ be an imaginary quadratic number field with ring of integers $\mathcal{O}_{-m}$, which we also just denote by $\mathcal{O}$. Consider the familiar action (we give an explicit formula for it in Lemma 4) of the group $\Gamma:=\mathrm{SL}_{2}(\mathcal{O}) \subset \mathrm{GL}_{2}(\mathbb{C})$ on hyperbolic three-space, for which we will use the upper-half space model $\mathcal{H}$.

As a set,

$$
\mathcal{H}=\{(z, \zeta) \in \mathbb{C} \times \mathbb{R} \mid \zeta>0\}
$$

Lemma 4 (Poincaré). If $\gamma=\left(\begin{array}{ll}a & b \\ c & d\end{array}\right) \in \mathrm{GL}_{2}(\mathbb{C})$, the action of $\gamma$ on $\mathcal{H}$ is given by $\gamma \cdot(z, \zeta)=$ $\left(z^{\prime}, \zeta^{\prime}\right)$, where

$$
\zeta^{\prime}=\frac{|\operatorname{det} \gamma| \zeta}{|c z-d|^{2}+\zeta^{2}|c|^{2}}, \quad z^{\prime}=\frac{(\overline{d-c z})(a z-b)-\zeta^{2} \bar{c} a}{|c z-d|^{2}+\zeta^{2}|c|^{2}}
$$

The Bianchi-Humbert theory $[\mathbf{5}, \mathbf{1 8}]$ gives a fundamental domain for the action of $\Gamma$ on $\mathcal{H}$, which we shall call the Bianchi fundamental polyhedron. It is a polyhedron in hyperbolic space up to the missing vertex $\infty$, and up to a missing vertex for each non-trivial ideal class if $\mathcal{O}_{-m}$ is not a principal ideal domain. We observe the following notion of strictness of the fundamental domain: the interior of the Bianchi fundamental polyhedron contains no two points which are identified by $\Gamma$. Swan [32] proves a theorem which implies that the boundary of the Bianchi fundamental polyhedron consists of finitely many cells.

\footnotetext{
†Observe that since we are not working within the adelic setting, we only consider Hecke operators which stabilize the connected components of the adelic symmetric space.
} 


\subsection{A cell complex for the Bianchi groups}

Swan further produces a concept for an algorithm to compute the Bianchi fundamental polyhedron. Such an algorithm has been implemented by Cremona [9] for the five cases where $\mathcal{O}_{-m}$ is Euclidean, and by his students Whitley [34] for the non-Euclidean principal ideal domain cases, Bygott [7] for a case of class number 2 and Lingham ([20], used in [11]) for some cases of class number 3, and finally Aranés [1] for arbitrary class numbers. Another algorithm based on this concept has been independently implemented in [23] for all Bianchi groups, and we make explicit use of the cell complexes it produces. Other results of the employed implementation are described in [24].

Definition 5. A pair of elements $(\mu, \lambda) \in \mathcal{O}^{2}$ is called unimodular if the ideal sum $\mu \mathcal{O}+\lambda \mathcal{O}$ equals $\mathcal{O}$.

The boundary of $\mathcal{H}$ is the Riemann sphere $\partial \mathcal{H}=\mathbb{C} \cup\{\infty\}$ (as a set), which contains the complex plane $\mathbb{C}$. The totally geodesic surfaces in $\mathcal{H}$ are the Euclidean vertical planes (we define vertical as orthogonal to the complex plane) and the Euclidean hemispheres centred on the complex plane.

Notation 6. Given a unimodular pair $(\mu, \lambda) \in \mathcal{O}^{2}$ with $\mu \neq 0$, let $S_{\mu, \lambda} \subset \mathcal{H}$ denote the hemisphere given by the equation $|\mu z-\lambda|^{2}+|\mu|^{2} \zeta^{2}=1$.

This hemisphere has centre $\lambda / \mu$ on the complex plane $\mathbb{C}$, and radius $1 /|\mu|$. Let

$$
\begin{aligned}
B:=\left\{(z, \zeta) \in \mathcal{H}: \text { the inequality }|\mu z-\lambda|^{2}+|\mu|^{2} \zeta^{2} \geqslant 1\right. \\
\left.\quad \text { is fulfilled for all unimodular pairs }(\mu, \lambda) \in \mathcal{O}^{2} \text { with } \mu \neq 0\right\} .
\end{aligned}
$$

Then $B$ is the set of points in $\mathcal{H}$ which lie above or on all hemispheres $S_{\mu, \lambda}$.

Lemma 7 [32]. The set $B$ contains representatives for all the orbits of points under the action of $\mathrm{SL}_{2}(\mathcal{O})$ on $\mathcal{H}$.

The action extends continuously to the boundary $\partial \mathcal{H}$, which is a Riemann sphere.

In $\Gamma:=\mathrm{SL}_{2}\left(\mathcal{O}_{-m}\right)$, consider the stabilizer subgroup $\Gamma_{\infty}$ of the point $\infty \in \partial \mathcal{H}$. Excluding the two cases $m=1$ and $m=3$ of Gaussian and Eisenstein integers, the latter group is given as

$$
\Gamma_{\infty}=\left\{ \pm\left(\begin{array}{ll}
1 & \lambda \\
0 & 1
\end{array}\right) \mid \lambda \in \mathcal{O}\right\}
$$

which performs translations by the elements of $\mathcal{O}$ with respect to the Euclidean geometry of the upper-half space $\mathcal{H}$.

Notation 8. A fundamental domain for $\Gamma_{\infty}$ in the complex plane (as a subset of $\partial \mathcal{H}$ ) is given by the rectangle

$$
D_{0}:= \begin{cases}\{x+y \sqrt{-m} \in \mathbb{C} \mid 0 \leqslant x \leqslant 1,0 \leqslant y \leqslant 1\}, & m \equiv 1 \text { or } 2 \bmod 4, \\ \left\{x+y \sqrt{-m} \in \mathbb{C} \mid \frac{-1}{2} \leqslant x \leqslant \frac{1}{2}, 0 \leqslant y \leqslant \frac{1}{2}\right\}, & m \equiv 3 \text { mod } 4 .\end{cases}
$$

And a fundamental domain for $\Gamma_{\infty}$ in $\mathcal{H}$ is given by

$$
D_{\infty}:=\left\{(z, \zeta) \in \mathcal{H} \mid z \in D_{0}\right\} .
$$

Definition 9. We define the Bianchi fundamental polyhedron as

$$
D:=D_{\infty} \cap B .
$$


We can check that the computed polyhedron is indeed a fundamental domain for $\Gamma$ using the following observation of Poincaré [22]: after a cell subdivision which makes the cell stabilizers fix the cells point-wise, the 2-cells ('faces') of the fundamental polyhedron appear in pairs $(\sigma, \gamma \cdot \sigma)$ with $\gamma \in \Gamma$ (so for every orbit of faces, we have exactly two representatives) such that with the orientation for which the lower side of the face $\sigma$ lies on the polyhedron, the upper side of $\gamma \cdot \sigma$ lies on the polyhedron. We induce a cell structure on $\mathcal{H}$ by the images under $\Gamma$ of the faces, edges and vertices of the Bianchi fundamental polyhedron.

\subsection{The Flöge cellular complex}

In order to obtain a cell complex with compact quotient space, we proceed in the following way due to Flöge [16]. The boundary of $\mathcal{H}$ is the Riemann sphere $\partial \mathcal{H}$, which, as a topological space, is made up of the complex plane $\mathbb{C}$ compactified with the cusp $\infty$. The totally geodesic surfaces in $\mathcal{H}$ are the Euclidean vertical planes (we define vertical as orthogonal to the complex plane) and the Euclidean hemispheres centered on the complex plane. The action of the Bianchi groups extends continuously to the boundary $\partial \mathcal{H}$. The cellular closure of the refined cell complex in $\mathcal{H} \cup \partial \mathcal{H}$ consists of $\mathcal{H}$ and $(\mathbb{Q}(\sqrt{-m}) \cup\{\infty\}) \subset(\mathbb{C} \cup\{\infty\}) \cong \partial \mathcal{H}$. The $\mathrm{SL}_{2}\left(\mathcal{O}_{-m}\right)$-orbit of a cusp $\lambda / \mu$ in $(\mathbb{Q}(\sqrt{-m}) \cup\{\infty\})$ corresponds to the ideal class $[(\lambda, \mu)]$ of $\mathcal{O}_{-m}$. It is well known that this does not depend on the choice of the representative $\lambda / \mu$. We extend the refined cell complex to a cell complex $\widetilde{X}$ by joining to it, in the case that $\mathcal{O}_{-m}$ is not a principal ideal domain, the $\mathrm{SL}_{2}\left(\mathcal{O}_{-m}\right)$-orbits of the cusps $\lambda / \mu$ for which the ideal $(\lambda, \mu)$ is not principal. We call the latter cusps the singular cusps. At the singular cusps, we equip $\widetilde{X}$ with the 'horoball topology' described in [16]. This simply means that the set of cusps, which is discrete in $\partial \mathcal{H}$, is located at the hyperbolic extremities of $\widetilde{X}$ : no neighborhood of a cusp, except the whole $\widetilde{X}$, contains any other cusp.

We retract $\widetilde{X}$ in the following, $\mathrm{SL}_{2}\left(\mathcal{O}_{-m}\right)$-equivariant, way. On the Bianchi fundamental polyhedron, the retraction is given by the vertical projection (away from the cusp $\infty$ ) onto its facets which are closed in $\mathcal{H} \cup \partial \mathcal{H}$. The latter are the facets which do not touch the cusp $\infty$, and are the bottom facets with respect to our vertical direction. The retraction is continued on $\mathcal{H}$ by the group action. It is proven in [15] that this retraction is continuous. We call the retract of $\widetilde{X}$ the Flöge cellular complex and denote it by $X$. So in the principal ideal domain cases, $X$ is a retract of the refined cell complex, obtained by contracting the Bianchi fundamental polyhedron onto its cells which do not touch the boundary of $\mathcal{H}$. In [27], it is checked that the Flöge cellular complex is contractible. Further details about the Flöge cellular complex and homological computations with it are described in [25].

\subsection{The spectral sequence}

Let $X$ be our Flöge complex constructed as above. Next we will consider the spectral sequence associated to the double complex $\operatorname{Hom}_{\mathbb{Z} \Gamma}\left(\Theta_{*}, C_{\mathbb{Z}}^{*}(X, M)\right)$, where $\Theta_{*}$ is the standard resolution of $\mathbb{Z}$ over $\mathbb{Z} \Gamma$ and $C^{*}(X, M)$ is the cellular co-chain complex of $X$ with $\mathbb{Z} \Gamma$-module coefficients $M$. We can (see [6, p. 164]) derive the first-quadrant spectral sequence

$$
E_{1}^{p, q}(M)=\bigoplus_{\sigma \in \Sigma_{p}} H^{q}\left(\Gamma_{\sigma} ; M\right) \Longrightarrow H^{p+q}(\Gamma ; M)
$$

where $\Sigma_{p}$ denotes the $\Gamma$-conjugacy classes of $p$-cells of $X$. Observe that $\Gamma_{\sigma}$ will be a finite group whose order is divisible only by 2 and/or 3 unless $\sigma$ is the class of a singular cusp, in which case $\Gamma_{\sigma}$ is a free Abelian group on two unipotent generators.

Assume that $M$ admits an additional module structure over a ring where 6 is invertible (in fact we are interested in the case where $M$ is a complex vector space). Then the finite ones among the higher cohomology groups of the $\Gamma_{\sigma}$ vanish. Thus, when there are no singular cusps (equivalently, when the class number of $\mathcal{O}$ is one), the spectral sequence concentrates on the 
row $q=0$ and stabilizes on the $E^{2}$-page. Otherwise, the spectral sequence concentrates on the rows $q=0,1,2$ and stabilizes at the $E^{3}$-page.

As we shall see below, the dimension of the module $H^{2}(\Gamma ; M)$, which we want to determine, is the same as the dimension of

$$
E_{2}^{2,0} \simeq E_{1}^{2,0} / \operatorname{Im}\left(d_{1}^{1,0}\right)
$$

where the differential $d_{1}^{1,0}$ is between

$$
E_{1}^{1,0} \simeq \bigoplus_{\sigma \in \Sigma_{1}} M^{\Gamma_{\sigma}} \longrightarrow \bigoplus_{\sigma \in \Sigma_{2}} M \simeq E_{1}^{2,0}
$$

The abutment of the spectral sequence gives us

$$
H^{2}(\Gamma ; M) \simeq E_{3}^{2,0} \oplus E_{3}^{0,2} .
$$

Here $E_{3}^{0,2} \simeq \bigoplus_{s} H^{2}\left(\Gamma_{s} ; M\right)$ where the summation is over $\Gamma$-classes of singular cusps $s$.

Moreover, $E_{3}^{2,0}=E_{2}^{2,0} / \operatorname{Im}\left(d_{2}^{0,1}\right)$ where the differential $d_{2}^{0,1}$ is between

$$
\bigoplus_{s \text { singular }} H^{1}\left(\Gamma_{s} ; M\right) \longrightarrow E_{2}^{2,0} .
$$

We determine the rank of this differential as follows.

Theorem 10 [31, Théorème 8]. Suppose that the coefficient module $M$ is equipped with a non-degenerate $\Gamma$-invariant $\mathbb{C}$-bilinear form. Then the rank of the map from $H^{1}(\Gamma ; M)$ to the disjoint sum of the $H^{1}\left(\Gamma_{s} ; M\right)$, induced by restriction from $H^{1}(\Gamma ; M)$ to $H^{1}\left(\Gamma_{s} ; M\right)$, equals half of the rank of the disjoint sum of the $H^{1}\left(\Gamma_{s} ; M\right)$.

The local topology of this map is studied in [26]. The image of this restriction-induced map can be identified with the image of the epimorphism in the short exact sequence of the spectral sequence's dévissage,

$$
0 \rightarrow E_{2}^{1,0} \longrightarrow H^{1}(\Gamma ; M) \longrightarrow \operatorname{ker} d_{2}^{0,1} \rightarrow 0 .
$$

Let us assume from now on that $M=E_{n}$ for some $n$. As we have seen in the proof of Proposition 1 , there is a perfect pairing on $M$, which is a non-degenerate $\Gamma$-invariant $\mathbb{C}$ bilinear form. So the theorem of Serre applies, and we obtain the following corollary. Note for this purpose that the proof of Proposition 1 shows that

$$
\operatorname{dim} H^{0}\left(\Gamma_{s} ; M\right)=\operatorname{dim} H^{2}\left(\Gamma_{s} ; M\right)=1 .
$$

When the cross-section of the cusp $s$ is a torus, we have

$$
\operatorname{dim} H^{1}\left(\Gamma_{s} ; M\right)=2 \cdot \operatorname{dim} H^{2}\left(\Gamma_{s} ; M\right)=2 .
$$

In the cases when $K$ is $\mathbb{Q}(i)$ or $\mathbb{Q}(\sqrt{-3})$, we have

$$
\operatorname{dim} H^{1}\left(\Gamma_{s} ; M\right)=0 .
$$

Corollary 11. The rank of the differential

is the number of non-trivial ideal classes.

$$
d_{2}^{0,1}: \bigoplus_{s \text { singular }} H^{1}\left(\Gamma_{s} ; M\right) \longrightarrow E_{2}^{2,0}
$$

REMARK 12. The above discussion implies that

$$
H^{2}(\Gamma ; M) \simeq\left(\bigoplus_{s \text { singular }} H^{2}\left(\Gamma_{s} ; M\right)\right) \oplus\left(E_{2}^{2,0} / \operatorname{Im}\left(d_{2}^{0,1}\right)\right),
$$

and the dimension of $H^{2}(\Gamma ; M)$ is the same as that of $E_{2}^{2,0}$. 


\subsection{The procedure of the computations}

We compute the representatives of faces in $E_{1}^{2,0}$ and the differential $d_{1}^{1,0}$ of our equivariant spectral sequence with trivial integer coefficients with the program Bianchi.gp [23]. The second author has implemented a MAGMA script that uses the cell stabilizers and identifications obtained with Bianchi.gp to compute the action on the coefficient module $M$ that we are interested in. We then deduce the term $E_{1}^{2,0}$ and the differential $d_{1}^{1,0}$ with respect to our coefficients. The quotient

$$
E_{2}^{2,0} \simeq E_{1}^{2,0} / \operatorname{Im}\left(d_{1}^{1,0}\right)
$$

now admits the dimension of $H^{2}(\Gamma ; M)$ by Remark 12 .

As linear algebra over number fields is more expensive compared to working over finite fields, we employ the following shortcut. Recall that by the universal coefficients theorem, the dimension of $H^{2}\left(\Gamma ; M\left(\mathbb{F}_{p}\right)\right)$ ('the mod $p$ dimension') is greater than or equal to the dimension of $H^{2}(\Gamma ; M(\mathbb{C})$ ) ('the complex dimension'). We start with computing the mod $p$-dimensions for primes $p \leqslant 200$. If we find a particular $p$ for which the $\bmod p$ dimension is equal to the lower bound of Finis-Grunewald-Tirao then we infer that the complex dimension is equal to the $\bmod p$ dimension. Note that by $[\mathbf{2 9}$, Proposition $3.2(\mathrm{~d})]$, this implies that $H^{2}(\Gamma ; M(\mathcal{O}))$ has no $p$-torsion. If this is not the case for the primes in our range, then we compute the complex dimension directly by computing $H^{2}(\Gamma ; M(K))$.

\subsection{Execution of the computations}

We applied the above described computations to a database of cell complexes for 186 Bianchi groups, which has been established on the computing clusters of the Weizmann Institute of Science, using over fifty processor-months. This database includes all the cases of ideal class numbers 3 and 5 , most of the cases of ideal class number 4 and all cases with the absolute value of the discriminant less than 500. Almost all of our dimension computations were carried out using the nodes of the computer clusters at the Universities of Duisburg-Essen and Luxembourg.

We are currently extending our computations to congruence subgroups (i.e. varying level) using the ICHEC computing cluster STOKES, and are soon going to release the results of those computations on the L-functions and Modular Forms DataBase (www.lmfdb.org).

Acknowledgements. We wish to thank John Cannon, John Cremona and Stephen S. Gelbart for useful discussions. We are grateful to Dan Yasaki who reworked his Magma program to compute for us the number field generated by the Hecke eigenvalues of the genuine weight two cuspidal Bianchi modular forms that we found for the field $\mathbb{Q}(\sqrt{-455})$. It is a pleasure to acknowledge our debt to Ulrich Görtz and Gabor Wiese for allowing us to use the computer nodes at the Institute for Experimental Mathematics of the University of Duisburg-Essen and the Mathematics Department of the University of Luxembourg respectively. Also, we are grateful to the Weizmann Institute of Science for providing the high performance computing clusters on which the database of cell complexes used for our calculations has been generated. The second author thanks the Algebra and Geometry Group of the Mathematics Department of the University of Barcelona for the post-doctoral fellowship under which he carried out most of his work that went into this paper. Moreover, he thanks the Mathematical Sciences Research Institute of the University of California and the Max Planck Institute for Mathematics for the wonderful hospitality that he received during his stays. Finally, we would like to thank Frank Calegari, Lassina Dembélé for their comments on the paper, and Aurel Page for computing the Hecke action on the weight $(1,1)$ cohomology for the field $\mathbb{Q}(\sqrt{-199})$. 


\section{References}

1. M. T. Aranés, 'Modular symbols over number fields', PhD Thesis, University of Warwick, 2010.

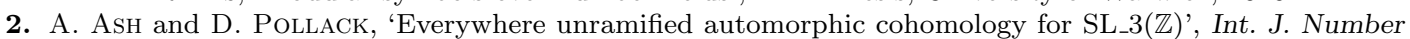
Theory 4 (2008) no. 4, 663-675.

3. A. Ash and G Stevens, 'Cohomology of arithmetic groups and congruences between systems of Hecke eigenvalues', J. Reine Angew. Math. 365 (1986) 192-220.

4. T. BERGER, 'Denominators of Eisenstein cohomology classes for GL_2 over imaginary quadratic fields', Manuscripta Math. 125 (2008) no. 4, 427-470.

5. L. Bianchi, 'Sui gruppi di sostituzioni lineari con coefficienti appartenenti a corpi quadratici immaginarî', Math. Ann. 40 (1892) no. 3, 332-412.

6. K. S. Brown, Cohomology of groups, Graduate Texts in Mathematics 87 (Springer, Berlin, 1982).

7. J. Вуяотт, 'Modular forms and modular symbols over imaginary quadratic fields', PhD Thesis, University of Exeter, 1998.

8. F. CAlegari and B Mazur, 'Nearly ordinary Galois deformations over arbitrary number fields', J. Inst. Math. Jussieu 8 (2009) no. 1, 99-177.

9. J. E. Cremona, 'Hyperbolic tessellations, modular symbols, and elliptic curves over complex quadratic fields', Compositio Math. 51 (1984) no. 3, 275-324.

10. J. E. Cremona, 'Abelian varieties with extra twist, cusp forms, and elliptic curves over imaginary quadratic fields', J. Lond. Math. Soc. (2) 45 (1992) no. 3, 404-416.

11. J. E. Cremona and M. P. Lingham, 'Finding all elliptic curves with good reduction outside a given set of primes', Exp. Math. 16 (2007) no. 3, 303-312.

12. K. DoI, H. HidA and H. IsHiI, 'Discriminant of Hecke fields and twisted adjoint $L$-values for GL(2)', Invent. Math. 134 (1998) no. 3, 547-577.

13. J. Elstrodt, F. Grunewald and J. Mennicke, 'On the group $\mathrm{PSL}_{2}(\mathbb{Z}[i])$ ', Number theory days, Exeter, 1980, London Mathematical Society Lecture Note Series 56 (Cambridge University Press, Cambridge, 1982) $255-283$.

14. T. Finis, F. Grunewald and P. Tirao, 'The cohomology of lattices in SL(2, C)', Exp. Math. 19 (2010) no. $1,29-63$.

15. D. FLÖGE, 'Zur Struktur der $\mathrm{PSL}_{2}$ über einigen imaginär-quadratischen Zahlringen', Dissertation, Johann-Wolfgang-Goethe-Universität, Fachbereich Mathematik, 1980.

16. D. FlÖGE, 'Zur Struktur der $\mathrm{PSL}_{2}$ über einigen imaginär-quadratischen Zahlringen', Math. Z. 183 (1983) no. 2, 255-279.

17. G. HARDER, 'On the cohomology of discrete arithmetically defined groups', Discrete subgroups of Lie groups and applications to moduli, Internat. Colloq., Bombay, 1973 (Oxford University Press, Oxford, 1975) 129-160.

18. G. Humbert, 'Sur la réduction des formes d'Hermite dans un corps quadratique imaginaire', C. R. Acad. Sci. Paris 16 (1915) 189-196.

19. N. KRÄMER, 'Beiträge zur Arithmetik imaginärquadratischer Zahlkörper', PhD Thesis, Math.-Naturwiss. Fakultät der Rheinischen Friedrich-Wilhelms-Universität Bonn; Bonn. Math. Schr., 1984.

20. M. Lingham, 'Modular forms and elliptic curves over imaginary quadratic fields', PhD Thesis, University of Nottingham, 2005.

21. A. PAGE, 'Computing arithmetic Kleinian groups', Preprint, 2012, arXiv:1206.0087 [math.NT].

22. H. Poincaré, 'Mémoire sur les groupes Kleinéens', Acta Math. 3 (1883) no. 1, 49-92.

23. A. D. RAhm, Bianchi.gp, Open source program (GNU general public license), validated by the CNRS, www.projet-plume.org/fiche/bianchigp part of the GP scripts library of Pari/GP Development Center, 2010 .

24. A. D. Rahm, 'Homology and K-theory of the Bianchi groups', C. R. Math. Acad. Sci. Paris 349 (2011) no. $11-12,615-619$.

25. A. D. RAHM, 'Higher torsion in the Abelianization of the full Bianchi groups', LMS J. Comput. Math., accepted (2013); http://hal.archives-ouvertes.fr/hal-00721690.

26. A. D. Rahm, 'On a question of Serre', C. R. Math. Acad. Sci. Paris 350 (2012) no. 15-16, 741-744.

27. A. D. RAHM and M. FuChS, 'The integral homology of PSL $\_2$ of imaginary quadratic integers with non-trivial class group', J. Pure Appl. Algebra 215 (2011) 1443-1472.

28. A. Scheutzow, 'Computing rational cohomology and Hecke eigenvalues for Bianchi groups', J. Number Theory 40 (1992) no. 3, 317-328.

29. M. H. ŞEnGüN, 'On the integral cohomology of Bianchi groups', Exp. Math. 20 (2011) no. 4, 487-505.

30. M. H. ŞEngün, 'Arithmetic aspects of Bianchi groups', Proceedings of Computations with Modular Forms, Heidelberg, 2011, to appear.

31. J.-P. SERre, 'Le problème des groupes de congruence pour SL(2)', Ann. of Math. (2) 92 (1970) $489-527$.

32. R. G. Swan, 'Generators and relations for certain special linear groups', Adv. Math. 6 (1971) 1-77.

33. R. TAYLOR, 'Representations of Galois groups associated to modular forms', Proceedings of the International Congress of Mathematicians, Vol. 1, 2 Zürich, 1994 (Birkhäuser, Basel, 1995) 435-442.

34. E. Whitley, 'Modular symbols and elliptic curves over imaginary quadratic fields', PhD Thesis, University of Exeter, 1990. 
35. G. WiESE, 'On the faithfulness of parabolic cohomology as a Hecke module over a finite field', J. Reine Angew. Math. 606 (2007) 79-103.

36. D. YASAKI, "Hyperbolic tessellations associated to Bianchi groups", Algorithmic Number Theory, Proceedings of 9th International Symposium ANTS-IX, Nancy, France, July 19-23, 2010 (Springer, Berlin, 2010).

\author{
Alexander D. Rahm \\ Department of Mathematics \\ National University of Ireland at \\ Galway \\ Ireland
}

Alexander.Rahm@nuigalway.ie

\author{
Mehmet Haluk Şengün \\ Mathematics Institute \\ University of Warwick \\ Coventry CV4 7AL \\ United Kingdom
}

M.H.Sengun@warwick.ac.uk 